\title{
THE MAGNETODYNAMIC MODEL OF QUASARS
}

\author{
L. M. OZERNoJ \\ (P.N. Lebedev Physical Institute, Academy of Sciences of the U.S.S.R., Moscow)
}

\section{Introduction}

Although the energy problem is one of the main difficulties of the cosmological interpretation of quasar red-shifts, there is no need to go beyond conventional physical laws in seeking an explanation. Various proposals (1-10) have been made which differ in important details. I believe a suitable basis for a theory of quasars to be a supermassive plasma configuration, the matter of which is in mostly regular motion in a magnetic field $(5,7)$. Such a magnetodynamic configuration I shall call a 'magnetoid'. On this model a quasar is a galaxy with a dense non-stellar nucleus, the magnetoid, which gives rise to the characteristic non-thermal luminosity. The mass of the quasar is determined by its stars while the parameters of the magnetoid depend on the stage of evolution or conditions of formation in a similar way to those of radiogalaxies or active nuclei of galaxies.

\section{Important Features of Quasars}

The most important features of quasars are, firstly, the exceptionally powerful continuum radiation, up to $10^{46}-10^{47} \mathrm{ergs}^{-1}$ and, secondly, the time variations of this radiation the rapid component of which indicates linear dimensions of no more than $10^{15}-10^{17} \mathrm{~cm}$. A statistical investigation of the optical variability of $3 \mathrm{C} 273$ reveals significant differences from a gaussian distribution, and it may be concluded (11) that the source must represent what is effectively a single body rather than a large number of independent events such as multiple supernova explosions.

The constancy of mean output of 3C 273 over nearly a century means that the source must be in quasi-stationary equilibrium. At the rate of $10^{47} \mathrm{erg} \mathrm{s}^{-1}$ for $3 \times 10^{5}$ years (a likely time-scale for a quasar) the energy required is $10^{60} \mathrm{erg}$. The mass involved is therefore not less than $10^{8}-10^{9} M_{\odot}$. If a plasma cloud of such a mass were initially in static thermal equilibrium it would radiate its internal energy within a few years and, without some source of energy, would contract rapidly as predicted by the theory of general relativity. If, however, the equilibrium is stationary rather than static and the plasma has regular internal motions in a magnetic field, then the timescale can be sufficiently extended to account for the observations. 


\section{Features of the Magnetodynamic Configuration}

In the quasi-stationary state, the main motion of the magnetoid matter takes the form of orderly circulation of the plasma in a regular inhomogeneous magnetic field. There is also likely to be magnetoturbulence on various scales. The equilibrium is quasi-stationary because of continuous outflow of matter from the surface as well as transport of angular momentum and magnetic field.

The magnetoid has two important properties which explain the main peculiarities of quasars:

(i) A powerful energy output from a small volume. On reaching the boundary of stable equilibrium the laminar motion in the magnetoid is apparently destroyed. In (5) and (7) consideration was given to the limiting situation where the 'break-down' of quasi-equilibrium occurs in a state of magnetoturbulence. For the simplest case when the magnetoturbulent energy $E_{\mathrm{mt}}$ is small compared with the thermal energy $E_{t}$, an analytical solution is possible. Instability occurs at a magnetoid radius of $R_{\mathrm{cr}} \approx 3 \cdot 5\left(3+\tau^{-1}\right) R_{\mathrm{g}}$, where $\tau=E_{\mathrm{mt}} / E_{\mathrm{t}}$ and $R_{\mathrm{g}}$ is a gravitational radius. If, e.g., $\tau=2 / 9$ (the maximum turbulent velocity being limited by the velocity of sound so that the damping of magnetoturbulence is small) then $R_{\mathrm{cr}} \approx 26 R_{\mathrm{g}}$, which is much less than in the static case. The energy corresponding to the critical state equals $\mathscr{E} \approx-0 \cdot 11$ $\left(3+\tau^{-1}\right)^{-2} M c^{2}$, and putting $\tau=2 / 9$ we obtain $|\mathscr{E}| \approx 2 \times 10^{-3} M c^{2}=4 \times 10^{51} M / M_{\odot}$ erg.

It should be stressed that the total output of energy is proportional to the mass. In the polytropic approximation the magnetoid lifetime of about $10^{6}$ years is determined by the thermal losses and is independent of the mass. If the mass does not exceed the value $M_{*} \approx 2 \times 10^{10}\left(3+\tau^{-1}\right)^{-2} M_{\odot}$, i.e. about $4 \times 10^{8} M_{\odot}$ if $\tau=2 / 9$, then nuclear reactions take place during the quasi-static contraction, providing an extra energy source. For large masses, however, with $M>M_{*}$, gravitational contraction turns out to be the main energy source.

(ii) Variability of the radiated energy. This is readily understood if the large-scale circular motion is inhomogeneous, anisotropic and, in particular, not axially symmetrical. The time variations will consist of a long-period component due to the circular motion and fast fluctuations associated with the turbulence and active flare processes. A comparison of the theory of these variations (12) with the light curve of 3C 273 over 80 years $(13, \mathbf{1 4})$ shows that the observations are in agreement with the idea of large-scale motion in the magnetic field of a quasar nucleus. This field may be thought of as providing 'magnetic rails' on which the circular motion proceeds with little damping.

\section{Observational Tests of the Magnetodynamic Model}

The model described above explains some of the observations and also predicts certain effects which may serve as observational tests (15). First of all, however, it is 
important to check the main consequences of the theory, i.e. the existence in quasar nuclei of strong magnetic fields and large-scale motion. The first may be tested by analysis of the synchrotron radiation of quasars. Such an analysis of the infrared radiation of $3 C 273(7,16)$ indicates a field strength of several hundred oersted - an unprecedented value for extragalactic sources but easily provided by the magnetodynamic model. The presence of large-scale motion is confirmed by the statistics of the optical continuum variations, as discussed already. In addition the regular motion will lead (12) to periodic alterations of the position angle of linearly polarized radiation. It is of interest that variations of position angle have recently been observed (17). The changes are non-monotonic and are, besides, irregular on a short time-scale. Such irregularities are to be expected as a consequence of the turbulent-convective motions. Frequent photoelectric observations of quasars such as $3 \mathrm{C} 446$ are vital for acquiring further information about the magnetoturbulence and important information about explosion processes in a magnetoid can be extracted from analysis of the radio variations $(\mathbf{2 0})$.

\section{The Magnetodynamic Model and the Origin of Quasars}

Magnetoids may result as a consequence of the contraction of an initially more rarefied medium and be dependent upon a suitable configuration of the magnetic field and rotation $(6,9)$. Formation may occur either in the intergalactic medium (especially in the past) or in the nuclei of existing galaxies.

Magnetoids in intergalactic space are likely to originate in rotating gas clouds of mass up to $10^{12} M_{\odot}$ which have a magnetic field roughly perpendicular to the rotation axis. In the central region, plasma will form a magnetoid while elsewhere fragmentation into star clusters will take place. Annihilation of oppositely directed magnetic fields near the rotation axis is expected to release magnetic energy explosively. The consequent shock wave will expand in each direction along the rotation axis and produce relativistic particles and plasma jets. Since the particle acceleration takes place at the shock front, this should be a region of strong radiation.

Magnetoids may also be formed in galactic nuclei from the interstellar gas concentrated there. In this case, however, the total mass does not exceed $10^{8} M_{\odot}$ and the energy output is less, corresponding to the phenomena in the nuclei of Seyfert galaxies.

\section{The Relation of Magnetoids to Star Formation}

The explosive release of energy on the formation of a magnetoid, and during its development, leads to the ejection of matter in the form of a plasma. On cooling, this plasma will fragment into stars (18), thus providing a new process for the formation of stars and star clusters (see Figure 1).

Before cooling and fragmentation the plasma will contain large numbers of relativ- 
istic particles and will radiate strongly, thus explaining the pairs of radio-sources associated with certain peculiar galaxies. Calculations indicate that the mass of plasma ejected in this way may reach that of small galaxies. The fragmented parts are then expected to have masses of the order of those of globular clusters, which may account for the unusual number of globular clusters in NGC 4486. This can be regarded as the physical realization of Ambarcumjan's idea (19) on star formation from

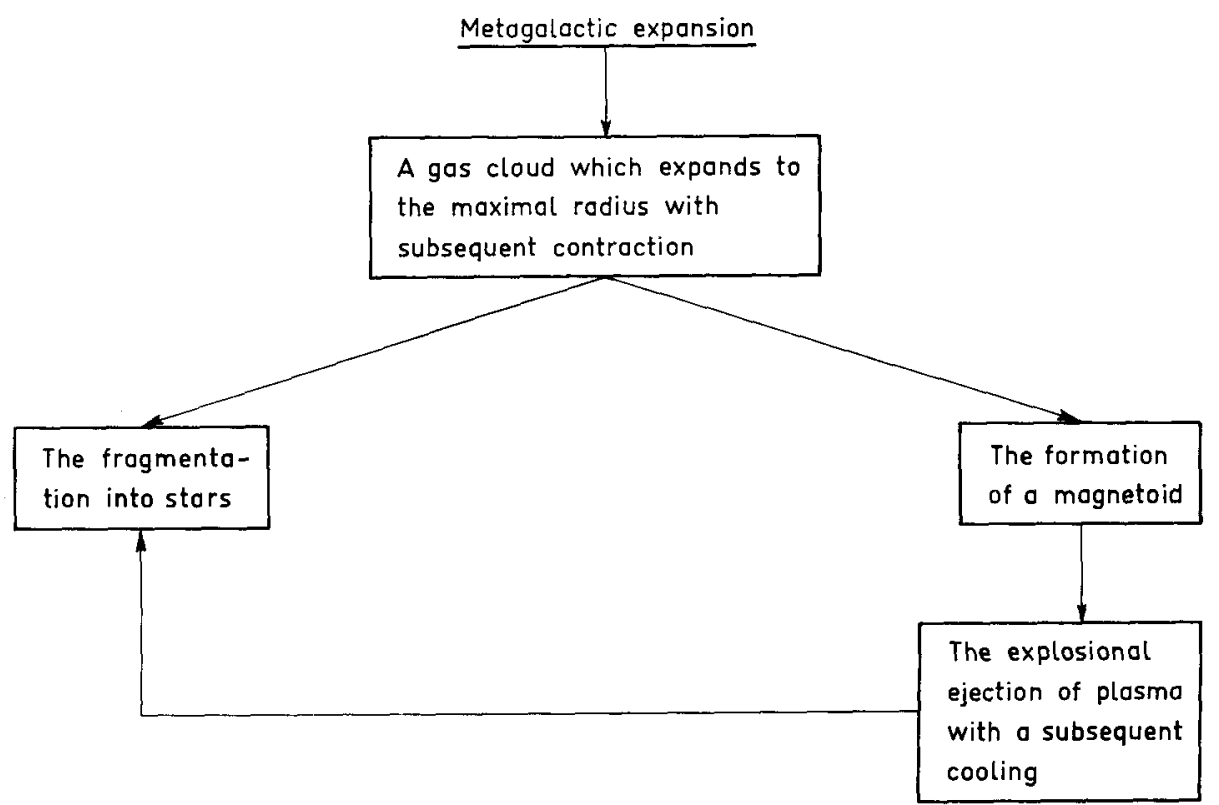

Fig. 1. The scheme of a dualistic way of star formation in a galaxy.

material denser than interstellar matter. The eruptional genesis of stars and stellar systems turns out, however, to be only a part of the common scheme of gravitational condensation, with the matter traversing from the rarefied dispersion phase into the dense structure phase (21) via the intermediate stage of a magnetoid.

\section{Conclusions}

The magnetodynamic model provides the necessary energy source for quasars and explains the principal observational data. It suggests that such varied objects as quasars, radio-galaxies and active nuclei of galaxies may have similar energy reservoirs, quantitatively different due to the different conditions giving rise to the magnetoids. It is hoped that a more detailed treatment will account for the differences between these phenomena. 


\section{References}

1. Fowler, W.A. (1964) Rev. mod. Phys., 36, 545; (1966) Astrophys. J., 144, 180.

2. Ginzburg, V.L., Ozernoj, L.M. (1964) Zu. eksp. teor. Fiz., 47, 1030.

3. Ozernoj, L.M. (1966) Soviet Phys. Dokl., 10, 581.

4. Layzer, D. (1965) Astrophys. J., 141, 837.

5. Ozernoj, L. M. (1967) in Proc. Symp. "Variable Stars and Stellar Evolution" (Moscow 1964), Nauka Publ. House, Moscow, pp. 140-155.

6. Ozernoj, L. M. (1967) ibid., pp. 13-39.

7. Ozernoj, L. M. (1966) Soviet Astr., 10, 241.

8. Sturrock, P. (1965) Nature, 205, 861; (1966) Nature, 211, 697.

9. Piddington, J.H. (1966) Mon. Not. R. astr. Soc., 133, 163.

10. Bisnovaty-Kogan, G.S., Zel'dovic, Ja. B., Novikov, I.D. (1967) Astr. Zu., 44, 525.

11. Gudzenko, L.I., Ozernoj, L. M., Certoprud, V.E. (1967) Nature, 215, 605.

12. Ozernoj, L. M., Certoprud, V.E. (1967) Astr. Zu., 44, 537.

13. Ozernoj, L. M., Certoprud, V.E. (1966) Soviet Astr., 10, 15.

14. Smith, H.J. (1965) in Quasi-Stellar Sources and Gravitational Collapse, Ed. by I. Robinson et al., Univ. of Chicago Press, Chicago, p. 221.

15. Ginzburg, V.L., Ozernoj, L. M. (1967) in Proc. of 3rdTexas Symp. on Relativistic Astrophysics, New York (in press).

16. Šklovskij, I.S. (1965) Soviet Astr., 9, 683.

17. Kinman, T.D., Lamla, E., Wirtanen, C.A. (1966) Astrophys. J., 146, 964.

18. Ozernoj, L. M. Astr. $Z u$. (in press).

19. Ambarcumjan, V.A. (1958) in La Structure et L'Evolution de L'Univers (Proc. of 11 th Solvay Conference), Ed. by R. Stoops, Brussels, p. 241.

20. Ozernoj, L. M., Sazonov, V.N. (1968) Nature (in press); Astr. $Z u$. (in press).

21. Ozernoj, L. M., Cernin, A.D. (1967) Astr. Zu., 44, 1131; Zu. eksp. teor. Fiz. (Pisa) (in press); Astr. $Z u .45$, (in press).

\section{DISCUSSION}

Cowling: What does a magnetoid look like?

Ozernoj: Unfortunately I cannot draw one. 